\title{
Comparative incidence and burden of respiratory viruses associated with hospitalization in adults in New York City
}

\author{
William Sieling ${ }^{1}$, Connor Goldman ${ }^{1}$, Matthew Oberhardt ${ }^{2}$, Matthew Phillips ${ }^{3}$, Lynn \\ Finelli $^{3}$, and Lisa Saiman ${ }^{1}$ \\ ${ }^{1}$ Columbia University Irving Medical Center \\ ${ }^{2}$ New York-Presbyterian Hospital \\ ${ }^{3}$ Merck \& Co Inc
}

December 15, 2020

\begin{abstract}
Background: Although the burden of influenza is well characterized, the burden of community-onset non-influenza respiratory viruses has not been systematically assessed. Understanding the severity and seasonality of non-influenza viruses, including human coronaviruses, will provide a better understanding of the overall disease burden from respiratory viruses that could better inform resource utilization for hospitals and highlight the value of preventative strategies, including vaccines. Methods: From October 2017 to September 2019, a retrospective study was performed in a pre-defined catchment area to estimate the population-based incidence of community-onset respiratory viruses associated with hospitalization. Included patients were $>18$ years old, resided in New York City, were hospitalized for $>24$ hours, and had a respiratory virus detected within 3 calendardays of admission. Disease burden was measured by hospital length of stay (LOS), intensive care unit (ICU) admissions, and in-hospital mortality and compared among those with laboratory-confirmed influenza versus those with laboratory-confirmed non-influenza viruses (human coronaviruses, parainfluenza viruses, respiratory syncytial virus, human metapneumovirus, and adenovirus). Results: During the study period, 4,232 eligible patients were identified of whom $50.9 \%$ were $>65$ years of age. For each virus, the population-based incidence was highest for those $>80$ years of age. When compared to those with influenza viruses detected, those with non-influenza respiratory viruses detected (combined) had higher population-based incidence, significantly more ICU admissions, and higher in-house mortality. Conclusions: The burden of non-influenza respiratory viruses for hospitalized adults is substantial. Prevention and treatment strategies are needed for non-influenza respiratory viruses, particularly for older adults.
\end{abstract}

\section{Comparative incidence and burden of respiratory viruses associated with hospitalization in adults in New York City}

Running title: Burden of respiratory viruses

William D. Sieling, $\mathrm{MPH}^{1,2}$; Connor R. Goldman, $\mathrm{MPH}^{1,3}$; Matthew Oberhardt, $\mathrm{PhD}^{4}$; Matthew Phillips, $\mathrm{MPH}^{5}$; Lyn Finelli, DrPH, MS ${ }^{5}$; Lisa Saiman, MD, MPH ${ }^{1,6}$

${ }^{1}$ Department of Pediatrics, Columbia University Irving Medical Center, New York, NY

${ }^{2}$ Current Address; University of Minnesota Medical School, Duluth, MN

${ }^{3}$ Current address: Center for Observational and Real-World Evidence, Merck \& Co., Inc., Kenilworth, NJ

${ }^{4}$ Value Institute, New York-Presbyterian Hospital, New York, NY

${ }^{5}$ Center for Observational and Real-World Evidence, Merck \& Co., Inc., Kenilworth, NJ 
${ }^{6}$ Department of Infection Prevention \& Control, NewYork-Presbyterian Hospital, New York, NY

Acknowledgements: This work was supported by a research grant from Merck \& Co., Inc., Kenilworth, NJ, USA.

Potential conflicts of interest: M. P. and L. F. Finelli are employees of Merck \& Co., Inc. and L.S. has received research funding from Merck \& Co., Inc. and served on an Advisory Board for Merck \& Co., Inc.

Corresponding author: Lisa Saiman, MD MPH

Columbia University Irving Medical Center

622 West $168^{\text {th }}$ Street PH4W-470

New York, New York 10032

ls5@cumc.columbia.edu 2123059446

\begin{abstract}
Background: Although the burden of influenza is well characterized, the burden of community-onset non-influenza respiratory viruses has not been systematically assessed. Understanding the severity and seasonality of non-influenza viruses, including human coronaviruses, will provide a better understanding of the overall disease burden from respiratory viruses that could better inform resource utilization for hospitals and highlight the value of preventative strategies, including vaccines.
\end{abstract}

Methods: From October 2017 to September 2019, a retrospective study was performed in a pre-defined catchment area to estimate the population-based incidence of community-onset respiratory viruses associated with hospitalization. Included patients were $>18$ years old, resided in New York City, were hospitalized for $>24$ hours, and had a respiratory virus detected within 3 calendar-days of admission. Disease burden was measured by hospital length of stay (LOS), intensive care unit (ICU) admissions, and in-hospital mortality and compared among those with laboratory-confirmed influenza versus those with laboratoryconfirmed non-influenza viruses (human coronaviruses, parainfluenza viruses, respiratory syncytial virus, human metapneumovirus, and adenovirus).

Results: During the study period, 4,232 eligible patients were identified of whom $50.9 \%$ were $>65$ years of age. For each virus, the population-based incidence was highest for those $>80$ years of age. When compared to those with influenza viruses detected, those with non-influenza respiratory viruses detected (combined) had higher population-based incidence, significantly more ICU admissions, and higher in-house mortality.

Conclusions: The burden of non-influenza respiratory viruses for hospitalized adults is substantial. Prevention and treatment strategies are needed for non-influenza respiratory viruses, particularly for older adults.

Keywords : influenza, human coronaviruses, parainfluenza viruses, respiratory syncytial virus, adenovirus

\title{
BACKGROUND
}

Respiratory viruses cause significant morbidity and mortality in adults, especially among frail older adults and those with chronic comorbid conditions [1,2]. While the burden of influenza viruses is best studied, non-influenza viruses such as respiratory syncytial virus (RSV), human metapneumovirus (hMPV), human coronaviruses $(\mathrm{CoV})$, adenoviruses $(\mathrm{AV})$, and parainfluenza viruses (PIV) are responsible for a substantial burden of illness in adults [3-6].

However, the population-based incidence and burden of community-onset non-influenza respiratory viruses associated with hospitalization in adults have not been systematically assessed. Previous approaches have utilized weekly laboratory-surveillance and syndromic surveillance associated with discharge data in statistical models to estimate rates of hospitalization and mortality associated with respiratory viruses $[4,7,8]$. Yet, these methods may lack precision as specific viruses associated with acute respiratory infections (ARIs) are not routinely laboratory-confirmed nor consistently reported in discharge records. Thus, the aims of this 
study were to estimate the population-based incidence of different laboratory-confirmed respiratory viruses in hospitalized adults and to describe the disease burden associated with different viruses measured by hospital length of stay (LOS), intensive care unit (ICU) admissions, and in-hospital mortality. These parameters were compared in patients with influenza versus patients with non-influenza respiratory viruses in efforts to provide an enhanced understanding of the overall disease burden from respiratory viruses and better inform resource utilization for hospitals and highlight the value of preventative strategies, including vaccines.

\section{METHODS}

\section{Study Design, Sites, and Subjects}

From October 2017 to September 2019, a retrospective study was performed to identify hospitalized adults who had respiratory viruses detected from nasopharyngeal swab specimens using a multiplex polymerase chain reaction (mPCR) assay (described below). Study sites are located in Northern Manhattan, are part of the same academically affiliated hospital system, and include three hospitals: a $\sim 750$-bed tertiary care hospital caring for adults, a 200-bed community hospital caring for adults, and a 250-bed children's hospital.

To estimate the population-based incidence of community-onset respiratory viruses associated with hospitalization, eligible patients were 18 years of age and older, resided in New York City, as defined by the zip code of their residence, were hospitalized for at least 24 hours, and had a respiratory virus detected within 3 calendar-days of admission. Patients were identified from the electronic health record (EHR). The Columbia University Irving Medical Center institutional review board approved this study with a waiver of informed consent.

\section{Viral Detection}

As part of the standard of care, patients with ARI symptoms who are being admitted are tested for respiratory viruses to inform appropriate transmission precautions and antiviral therapy for influenza. The study sites use the FilmArray Respiratory Panel (BioFire Diagnostics, Inc., Salt Lake City, UT) which detects influenza (types A H3, A H1, B), PIV types 1-4, RSV, hMPV, AV, rhinovirus/enterovirus (RV/EV), and human coronaviruses (CoV types 229E, HKU1, NL63, OC43).

\section{Data Analysis}

Two one-year periods, October 2017 to September 2018 (year 1) and October 2018 to September 2019 (year 2 ), were studied to assess seasonal differences in population-based incidence and clinical burden for the respiratory viruses detected. To calculate population-based incidence per 100,000 persons for each virus, the number of adults with a laboratory-confirmed respiratory virus was divided by the adjusted 2010 U.S. Census population estimate for the catchment area. Population-based incidence and $95 \%$ confidence intervals were calculated overall and for four age strata: 18-49, 50-64, 65-79, and $>80$ years of age [9]. If more than one virus was detected in a single specimen, each virus contributed to the respective incidence calculation. The population estimate was adjusted by the hospitals' percent market share for zip codes as determined by the New York Statewide Planning and Research Cooperative System (SPARCS) [10]. To improve the incidence estimate's reliability, the catchment area was defined as the eight zip codes in which the hospitals had $>60 \%$ market share $(10032,10033,10034,10040,10452,10453,10463$, and 10471).

To assess the clinical burden of specific viruses, the following outcomes associated with each virus were determined: the median hospital LOS, the proportion of patients who had an ICU admission, the ICU LOS, and in-hospital all-cause mortality. Time spent in the emergency department prior to being admitted to an inpatient unit was included in LOS calculations. If more than one virus was detected in a single specimen, each virus contributed to the respective median LOS, ICU admission and ICU LOS, and in-hospital mortality which was determined from recorded death notes in the EHR. Two sample tests of proportions were used to compare outcomes (ICU admissions and mortality) between each virus and all others (e.g., adenovirus vs. all other viruses). Median hospital LOS and median ICU LOS were compared using the Mann-Whitney test for each virus vs. all others. 
The population-based incidence and clinical burden of influenza viruses (combined A H1, A H3, and B) were compared with those of non-influenza viruses (combined CoV229E, CoVHKU1, CoVNL63, CoVOC43, PIV types 1-4, RSV, hMPV, and AV) using the two sample test of proportions. RV/EV were excluded from the comparative analyses of the burden of influenza vs. non-influenza viruses. Patients with co-detections of influenza and non-influenza viruses were excluded from these comparative analyses.

\section{RESULTS}

\section{Study Population}

During the study period, 4,232 adults were hospitalized with a laboratory-confirmed respiratory virus and met the community-onset case definition. Testing for a respiratory virus occurred on hospital day 1 for $90.7 \%$ of patients, on hospital day 2 for $5.0 \%$ of patients, on hospital day 3 for $2.0 \%$ of patients, and $2.3 \%$ of patients had testing performed in ambulatory settings affiliated with the hospitals.

Selected demographic characteristics and the distribution of the zip codes of patients are shown in Table 1. Demographic characteristics were similar in year 1 and year $2 ; 50.9 \%$ of patients were $>65$ years of age and $23.5 \%$ of patients were $>80$ years of age. Overall, $2,447(57.8 \%)$ of patients resided in zip codes that represented $>60 \%$ of the hospital's market share.

\section{Viruses Detection}

The proportion of patients who had specific viruses detected is shown in Table 1. Overall, 904 patients had influenza detected, 1,667 had RV/EV detected, and 2,551 had non-influenza, non-RV/EV respiratory viruses detected. The relative proportion of each virus was similar in year 1 vs. year 2 with the exception of the influenza viruses (A H1, A H3, and B) and the human coronavirus types. Influenza A H3 and influenza B were most common in year 1, while influenza A H1 and influenza A H3 were most common in year 2. The cumulative proportion of all coronaviruses was similar in both years, but seasonal differences occurred in the relative proportions of CoV229E, CoVHKU1, and CoVOC43 as illustrated in Figure 1. In year 1, CoVHKU1 was most common and had a sharp winter peak. In year 2, CoV229E peaked in winter, but continued to be detected throughout the spring.

Overall, co-detection of two or more respiratory viruses occurred in 251 patients. In year 1, $116(5.7 \%)$ of 2,043 patients had co-detection and RV/EV was detected in $46(39.7 \%)$ of these 116 co-detections. In year 2, $135(6.2 \%)$ of 2,189 patients had co-detections and RV/EV was detected in $72(53.3 \%)$ of these 135 co-detections. Among the 251 total co-detections, an Influenza virus and a non-influenza virus were detected in $67(26.7 \%)$.

\section{Population-based Incidence}

The population-based incidence for each virus overall and by age strata for year 1 and year 2 are shown in Figures 2a and 2b, respectively. Each year, RV/EV had the highest overall population-based incidence estimates (in year 1, 173 hospitalizations and in year 2, 204 hospitalizations per 100,000 persons). After $\mathrm{RV} / \mathrm{EV}$, in year 1, influenza A H3 had the highest overall incidence (69 hospitalizations per 100,000 persons) followed by the combined CoV types (65 hospitalizations per 100,000 persons) and in year 2, the combined $\mathrm{CoV}$ types had the highest overall incidence (91 hospitalizations per 100,000 persons) followed by influenza A H1 (52 hospitalizations per 100,000 persons).

Incidence increased with age for all viruses for both years and those $>80$ years of age consistently had the highest incidence. The highest population-based incidence in those $>80$ years of age for specific viruses was 660 hospitalizations per 100,000 persons for influenza A H3 in year 1 and 535 hospitalizations per 100,000 persons for the four combined human coronaviruses in year 2. Following RV/EV, the combined CoV types had the highest incidence estimates in both years among patients 18-49 and 50-64 years of age.

In year 1, the combined population-based incidence of all influenza viruses (A H1, A H3, and B) for all age strata was significantly lower than the combined population-based incidence of the non-influenza respiratory viruses including CoV types, RSV, PIV types, hMPV, and AV (125 vs. 208 hospitalizations per 100,000 
persons, respectively, $\mathrm{p}<0.001)$. Similarly, in year 2 , the combined population-based incidence of influenza viruses was significantly lower than that of these non-influenza respiratory viruses (96 vs. 244 hospitalizations per 100,000 persons, respectively, $\mathrm{p}<0.001$ ).

\section{Clinical Burden associated with Different Viruses}

The median LOS, the proportion of subjects admitted to an ICU, the ICU LOS, and in-hospital mortality for each virus are shown in Table 2 . As the burden was similar in each year, data for both years were combined. The overall median LOS was 4.2 days (interquartile range [IQR] 2.3, 7.8 days) and similar for each virus. Overall, $14.4 \%$ of patients had an ICU admission with a median ICU LOS of 4.0 days (IQR 2.1, 8.4 days). Compared to other viruses, CoV229E $(\mathrm{p}=0.01)$ and $\mathrm{AV}(\mathrm{p}=0.02)$ were each associated with a significantly larger proportion of ICU admissions and AV was associated with the longest ICU LOS ( $\mathrm{p}=0.03)$. Of the 225 ICU admissions associated with RV/EV, 19 (8.4\%) had another virus co-detected. Four percent of patients died during hospitalization. RV/EV was associated with $31.8 \%$ of deaths and CoV229E was associated with the highest mortality rate $(12.3 \%, \mathrm{p}<0.001)$. The median LOS among those who died was 9.7 days (IQR $5.0,19.8$ days) and was longest for $\mathrm{AV}$ (38.0 days, $\mathrm{p}=0.03)$.

Those with RV/EV ( $n=1,667$ patients) and those with influenza/non-influenza co-detection ( $\mathrm{n}=67$ patients) were excluded from the analysis comparing the burden of influenza viruses (784 patients) to that of the noninfluenza viruses (1,714 patients). Compared to patients with influenza, a significantly higher proportion of patients with the non-influenza viruses CoV, RSV, PIV, hMPV, and AV were admitted to the ICU (11.0\% vs. $16.7 \%$, respectively, $\mathrm{p}=0.002)$. Compared to patients with influenza, a significantly higher proportion of patients with the non-influenza respiratory viruses died $(3.2 \%$ and $5.2 \%$, respectively, $\mathrm{p}=0.025)$.

\section{DISCUSSION}

This study provides further insights for the population-based incidence of laboratory-confirmed respiratory viruses associated with hospitalization in adults. Our findings may provide more precise estimates of the incidence of non-influenza respiratory viruses than previous studies which have often relied on diagnostic codes or syndromic surveillance, rather than systematic laboratory testing with a multiplex-PCR assay, and thus, may have underestimated the case burden [11]. We also evaluated the incidence of respiratory viruses in the subset of patients $>80$ years of age and found that the incidence in this oldest age strata was consistently more than double that of patients 65-79 years of age. These findings suggest that the impact of non-influenza respiratory viruses is likely underappreciated in the absence of systematic testing of older adults hospitalized with acute respiratory signs and symptoms. Furthermore, lack of diagnostic testing could lead to lack of appropriate infection prevention and control and potential nosocomial transmission.

We found that influenza viruses predictably exhibited a high seasonal incidence with different types each year. The burden of non-influenza respiratory viruses was potentially higher than that of influenza viruses. Noninfluenza respiratory viruses collectively had a higher incidence than that of influenza viruses collectively, although it is likely that the influenza vaccine reduced hospitalizations and mortality, particularly when there was a good match between the vaccine and circulating influenza strains [12]. We found that 2.8 times more patients were hospitalized with non-influenza viruses (excluding RV/EV) than with influenza viruses. Overall, the proportion of patients admitted to an ICU was significantly higher for non-influenza than for influenza viruses. Mortality was also significantly higher for non-influenza respiratory viruses. Notably, our analysis of the collective burden non-influenza viruses could be perceived as an underestimate as we excluded RV/EV, which was the most commonly detected virus, as similarly described by others [13]. We chose to exclude RV/EV from the analysis of the collective burden because RV/EV were the most commonly co-detected viruses and the mPCR assay used cannot distinguish rhinovirus from enterovirus nor unique subtypes. Furthermore, while RV/EV are associated with hospitalizations for ARIs [14] and exacerbations of underlying cardiac and pulmonary comorbidities [13-15], prolonged viral shedding is also well described [16] which could decrease the causal relationship of detecting these viruses with burden.

Comparison of our findings with other reports is challenging due to different methodologies including case finding and study population. For example, past studies exclusively examined older adults $>65$ years old and 
high-risk adults with congestive heart failure and chronic obstructive pulmonary disorder [6]. Furthermore, much of the morbidity and mortality associated with non-influenza respiratory viruses has been described in adult long-term care facilities experiencing outbreaks associated with high attack rates and high death rates $[17,18]$. Nonetheless, our incidence estimates of influenza hospitalizations were comparable to the Centers for Disease Control and Prevention (CDC) national estimates for both years [19, 20]. The current study's rate of ICU admissions from RSV (16.1\%) was similar to that described in previous prospective studies of RSV by Widmer and colleagues (10\%) [3] and Falsey and colleagues (15\%) [6]. The current study's ICU admission rate of $11.5 \%$ associated with influenza was also similar to that previously described (6\% [3], $12 \%$ $[6])$ as was the $16.5 \%$ rate associated with HMPV (13\% [3]).

Some of our observations for the human coronaviruses merit further study as, to our knowledge, these findings have not been previously reported. We found a high burden associated with CoV 229E and CoV HKU1 as these (in addition to AV) had the highest rates of ICU admission and highest mortality rate. Among all the viruses studied, the combined CoV types had the highest incidence estimates for patients 18-49 and 50-64 years of age for both years. We found similar seasonality among the human coronavirus types as has been reported by the Centers for Disease Control and Prevention (CDC) and National Respiratory and Enteric Virus Surveillance System NREVSS [19-21]. However, the most common CoV type found by laboratories participating in NREVSS in 2018-2019 was CoVOC43, while we found CoV229E and CoVOC43 to be the most common types that season. While surveillance reports from the NREVSS provide trends for and seasonality of respiratory viruses, these reports lack patient-level characteristics to inform age-related trends or patient outcomes. It remains to be seen if seasonal variations in the human coronavirus will impact the epidemiology of the COVID-19 pandemic or be a source of false positive serologic results for SARS-CoV-2 or even if SARS-CoV-2 could eventually become a seasonal virus.

This study has limitations. It was performed at a university affiliated tertiary care referral center in New York City which limits generalizability. We did not consider the impact of influenza vaccinations on hospitalization rate. Viral detections may not have represented acute infection as mPCR does not distinguish between viable and non-viable virus. Case detection may have been decreased if not all patients were tested for respiratory viruses, particularly outside influenza season. However, the study period aligns with a prospective surveillance study of RSV in hospitalized adults conducted in the same facilities in which we found that nearly all hospitalized patients meeting ARI criteria during the respiratory viral season, October to April, were tested by treating clinicians [22]. Finally, while the case definition required viral detection within three days of admission, we did not measure the burden of illness in cases that were acquired during hospitalization or in patients transferred to our hospital who had been tested at outside facilities.

In conclusion, the burden of non-influenza respiratory viruses is substantial, particularly for older adults who lack durable immunity for respiratory viruses and frequently have comorbid conditions that increase their risk of severe disease. Thus, prevention and treatment strategies for non-influenza respiratory viruses, including effective vaccines, are needed. Future research should further assess the clinical impact of specific human coronaviruses and the potential impact of specific coronavirus types on the epidemiology and impact of SARS-CoV-2.

\section{REFERENCES}

1. Blackburn R, Zhao H, Pebody R, Hayward A, Warren-Gash C. Laboratory-confirmed respiratory infections as predictors of hospital admission for myocardial infarction and stroke: time-series analysis of English data for 2004-2015. Clin Infect Dis, $2018 ; 67: 8-17$.

2. Mohan A, Chandra S, Agarwal D, et al. Prevalence of viral infection detected by PCR and RT-PCR in patients with acute exacerbation of COPD: a systematic review. Respirol, 2010 ;15:536-542.

3. Widmer K, Zhu Y, Williams JV, Griffin MR, Edwards KM, Keipp Talbot H. Rates of hospitalizations for respiratory syncytial virus, human metapneumovirus, and influenza virus in older adults. J Infect Dis,2012 
;206:56-62.

4. van Asten L, van den Wijngaard C, van Pelt W, et al. Mortality attributable to 9 common infections: significant effect of influenza A, respiratory syncytial virus, influenza B, norovirus, and parainfluenza in elderly persons. J Infect Dis, $2012 ; 206: 628-639$.

5. Lim YK, Kweon OJ, Kim HR, Kim TH, Lee MK. Clinical features, epidemiology, and climatic impact of genotype-specific human metapneumovirus infections: Long-term surveillance of hospitalized patients in South Korea. Clin Infect Dis, 2020 ;70:2683-2694.

6. Falsey AR, Hennessey PA, Formica MA, Cox C, Walsh EE. Respiratory syncytial virus infection in elderly and high-risk adults. N Engl J Med,2005 ;352:1749-1759.

7. Thompson WW, Shay DK, Weintraub E, et al. Mortality associated with influenza and respiratory syncytial virus in the United States. JAMA,2003;289:179-186.

8. Pitman RJ, Melegaro A, Gelb D, Siddiqui MR, Gay NJ, Edmunds WJ. Assessing the burden of influenza and other respiratory infections in England and Wales. J Infect, 2007 ;54:530-538.

9. Rothman KJ, Greenland S. Modern epidemiology. 3rd ed. Philadelphia: Lippincott Williams \& Wilkins, 2008 .

10. Statewide planning and Research Cooperative System. 2019 ; Available from: https://www.health.ny.gov/statistics/sparcs. Accessed November 13, 2020.

11. Pastula ST, Hackett J, Coalson J, et al. Hospitalizations for respiratory syncytial virus among adults in the United States, 1997-2012. Open Forum Infect Dis, 2017 ;4:ofw270.

12. Nordin J, Mullooly J, Poblete S, et. al. Influenza vaccine effectiveness in preventing hospitalizations and deaths in persons 65 years or older in Minnesota, New York, and Orgen: data from 3 health plans. J Infect Dis, $2001 ; 184: 665-670$.

13. Nicholson KG, Kent J, Hammersley V, Cancio E. Risk factors for lower respiratory complications of rhinovirus infections in elderly people living in the community: prospective cohort study. Brit Med J,1996; 313:1119-1123.

14. Pierangeli A, Scagnolari C, Selvaggi C, et al. Rhinovirus frequently detected in elderly adults attending an emergency department. J Med Virol, 2011 ;83:2043-2047.

15. Teichtahl H, Buckmaster N, and Pertnikovs E. The incidence of respiratory tract infection in adults requiring hospitalization for asthma. Chest, $1997 ; 112: 591-596$.

16. Peltola V, Waris M, Kainulainen L, Kero J, Ruuskanen O. Virus shedding after human rhinovirus infection in children, adults and patients with hypogammaglobulinaemia. Clin Microbiol Infect,2013;19:E322-7.

17. Kodama F, Nace DA, Jump RLP. Respiratory Syncytial Virus and Other Noninfluenza Respiratory Viruses in Older Adults. Infect Dis Clin North Am, 2017 ;31:767-790.

18. Ellis SE, Coffey CS, Mitchel Jr EF, Dittus RS, Griffin MR. Influenza- and respiratory syncytial virusassociated morbidity and mortality in the nursing home population. J Am Geriatr Soc,2003 ;51:761-767.

19. Garten R, Blanton L, Abd Elal AI, et al. Update: Influenza activity in the United States during the 2017-18 season and composition of the 2018-19 influenza vaccine. MMWR Morb Mortal Wkly Rep,2018 ;67:634-642.

20. Xu X, Blanton L, Abd Elal AI, et al. Update: Influenza activity in the United States during the 2018-19 Season and composition of the 2019-20 influenza vaccine. MMWR Morb Mortal Wkly Rep,2019;68:544-551.

21. Killerby ME, Biggs HM, Haynes A, et al. Human coronavirus circulation in the United States 2014-2017. J Clin Virol,2018;101:52-56. 
22. Branche A, Saiman L, Walsh EE, et al. Incidence of respiratory syncytial virus infection among hospitalized adults, 2017-2019. In: IDWeek 2019. Washington, D.C. Open Forum Infect Dis,2019;6:S7-S8.

Table 1: Selected Characteristics of Patients and Detected Respiratory Viruses

\begin{tabular}{|c|c|c|c|}
\hline & $\begin{array}{l}\text { Year } 1 \text { October } 2017 \\
\text { - September } 2018 \\
\mathrm{~N}=2043\end{array}$ & $\begin{array}{l}\text { Year } 2 \text { October } 2018 \\
\text { - September } 2019 \\
\mathrm{~N}=2189\end{array}$ & Total $\mathrm{N}=4232$ \\
\hline \multicolumn{4}{|l|}{ Demographic } \\
\hline \multicolumn{4}{|l|}{ Characteristics } \\
\hline \multicolumn{4}{|l|}{ Age in years, n (\%) } \\
\hline $18-49$ & $455(22.3 \%)$ & $526(24.0 \%)$ & $981(23.2 \%)$ \\
\hline $50-64$ & $542(26.5 \%)$ & $555(25.4 \%)$ & $1097(25.9 \%)$ \\
\hline $65-79$ & $534(26.14 \%)$ & $626(28.6 \%)$ & $1160(27.4 \%)$ \\
\hline$>80$ & $512(25.1 \%)$ & $482(22.0 \%)$ & $994(23.5 \%)$ \\
\hline Mean age (range) & $63.5(18-110)$ & $62.3(18-104)$ & $62.8(18-110)$ \\
\hline Female, n (\%) & $1144(56.0 \%)$ & $1247(57.0 \%)$ & $2391(56.5 \%)$ \\
\hline \multicolumn{4}{|l|}{ Hospital, n (\%) } \\
\hline A & $1322(64.7 \%)$ & $1526(69.7 \%)$ & $2848(67.3 \%)$ \\
\hline B & $644(31.5 \%)$ & $583(26.6 \%)$ & $1227(29.0 \%)$ \\
\hline $\mathrm{C}$ & $77(3.8 \%)$ & $80(3.7 \%)$ & $157(3.7 \%)$ \\
\hline $\begin{array}{l}\text { Reside in zip codes, } \mathrm{n} \\
(\%)>60 \% \text { market share }\end{array}$ & $1181(57.8 \%)$ & $1266(57.8 \%)$ & $2447(57.8 \%)$ \\
\hline $\begin{array}{l}\text { Reside in zip codes, } \mathrm{n} \\
(\%)<60 \% \text { market share }\end{array}$ & $862(42.2 \%)$ & $923(42.2 \%)$ & $1785(42.2 \%)$ \\
\hline \multicolumn{4}{|l|}{$\begin{array}{l}\text { Respiratory viruses } \\
\text { detected, }^{\mathrm{c}} \mathrm{n}(\%)\end{array}$} \\
\hline Influenza (all) & $503(23.3 \%)$ & $401(17.3 \%)$ & $904(20.2 \%)$ \\
\hline Influenza type AH3 & $270(12.5 \%)$ & $161(6.9 \%)$ & $431(9.6 \%)$ \\
\hline Influenza type AH1 & $48(2.2 \%)$ & $215(9.3 \%)$ & $263(5.9 \%)$ \\
\hline Influenza type B & $173(8.0 \%)$ & $14(0.6 \%)$ & $187(4.2 \%)$ \\
\hline Influenza A equivocal & $12(0.6 \%)$ & $11(0.5 \%)$ & $23(0.5 \%)$ \\
\hline Rhinovirus/Enterovirus & $765(35.5 \%)$ & $902(38.8 \%)$ & $1667(37.2 \%)$ \\
\hline $\begin{array}{l}\text { Human Coronavirus } \\
\text { (all) }\end{array}$ & $277(12.8 \%)$ & $366(15.7 \%)$ & $643(14.4 \%)$ \\
\hline Coronavirus type $229 \mathrm{E}$ & $20(0.9 \%)$ & $126(5.4 \%)$ & $146(3.3 \%)$ \\
\hline $\begin{array}{l}\text { Coronavirus type } \\
\text { HKU1 }\end{array}$ & $119(5.5 \%)$ & $30(1.3 \%)$ & $149(3.3 \%)$ \\
\hline Coronavirus type NL63 & $68(3.2 \%)$ & $59(2.5 \%)$ & $127(2.8 \%)$ \\
\hline Coronavirus type OC43 & $70(3.2)$ & $151(6.5 \%)$ & $221(4.9 \%)$ \\
\hline $\begin{array}{l}\text { Respiratory syncytial } \\
\text { virus }\end{array}$ & $198(9.2 \%)$ & $243(10.5 \%)$ & $441(9.8 \%)$ \\
\hline $\begin{array}{l}\text { Parainfluenza viruses } \\
1-4\end{array}$ & $193(8.9 \%)$ & $175(7.5 \%)$ & $368(8.2 \%)$ \\
\hline $\begin{array}{l}\text { Human } \\
\text { metapneumovirus }\end{array}$ & $182(8.4 \%)$ & $181(7.8 \%)$ & $363(8.1 \%)$ \\
\hline Adenovirus & $39(1.8 \%)$ & $54(2.3 \%)$ & $93(2.1 \%)$ \\
\hline
\end{tabular}

${ }^{\text {a }}$ Represents eight unique zip codes used for population-based incidence estimate.

${ }^{\mathrm{b}}$ Represents 130 and 133 unique zip codes in Year 1 and Year 2, respectively. 
'Includes specimens with more than one virus detected.

Table 2: Morbidity and Mortality Associated with Detection of Different Respiratory Viruses

\begin{tabular}{|c|c|c|c|c|c|c|}
\hline Virus & $\begin{array}{l}\text { Patients } \mathbf{n} \\
(\%)\end{array}$ & $\begin{array}{l}\text { Median } \\
\text { LOS, days } \\
\text { (IQR) }\end{array}$ & $\begin{array}{l}\text { ICU } \\
\text { Admission } \mathbf{n} \\
(\%)\end{array}$ & $\begin{array}{l}\text { ICU Median } \\
\text { LOS, days } \\
\text { (IQR) }\end{array}$ & $\begin{array}{l}\text { Crude } \\
\text { Mortality } \mathbf{n} \\
(\%)\end{array}$ & $\begin{array}{l}\text { Median LOS } \\
\text { of patients } \\
\text { who died, } \\
\text { days (IQR) }\end{array}$ \\
\hline $\begin{array}{l}\text { All } \\
\text { influenza }\end{array}$ & $904(20.2 \%)$ & $3.9(2.1,7.1)$ & $104(11.5)$ & $4.3(2.2,8.0)$ & $29(3.3)$ & $\begin{array}{l}8.7(5.5 \\
15.7)\end{array}$ \\
\hline Flu A (H3) & $431(9.6 \%)$ & $4.0(2.2,7.1)$ & $47(10.9)$ & $3.8(2.2,6.8)$ & $15(3.5)$ & $\begin{array}{l}6.2(5.1 \\
19.9)\end{array}$ \\
\hline Flu A (H1) & $263(5.9 \%)$ & $3.4(2.1,6.9)$ & $42(16.0)$ & $5.5(2.2,9.9)$ & $9(3.4)$ & $\begin{array}{l}8.5(8.3 \\
19.8)\end{array}$ \\
\hline Flu B & $187(4.2 \%)$ & $4.2(2.1,7.5)$ & $15(8.0)$ & $3.5(2.3,6.2)$ & $6(3.2)$ & $\begin{array}{l}10.9(10.0, \\
14.2)\end{array}$ \\
\hline $\mathrm{RV} / \mathrm{EV}$ & $\begin{array}{l}1667 \\
(37.2 \%)\end{array}$ & $4.1(2.3,7.6)$ & $225(13.5)$ & $3.8(2.1,7.7)$ & $54(3.2)$ & $\begin{array}{l}6.6(4.2 \\
18.4)\end{array}$ \\
\hline $\mathrm{CoV} 229 \mathrm{E}$ & $146(3.3 \%)$ & $4.4(2.3,8.1)$ & $32(21.9)$ & $\begin{array}{l}3.6(1.7 \\
10.8)\end{array}$ & $18(12.3)$ & $\begin{array}{l}11.0(5.5 \\
24.2)\end{array}$ \\
\hline CoVHKU1 & $149(3.3 \%)$ & $4.3(2.3,8.7)$ & $29(19.5)$ & $3.7(1.9,7.8)$ & $14(9.4)$ & $\begin{array}{l}7.8(3.8 \\
12.1)\end{array}$ \\
\hline CoVNL63 & $127(2.8 \%)$ & $5.1(3.0,8.9)$ & $26(20.5)$ & $3.3(2.0,7.6)$ & $5(3.9)$ & $\begin{array}{l}13.2(11.3, \\
24.5)\end{array}$ \\
\hline CoVOC43 & $221(4.9 \%)$ & $4.0(2.4,7.8)$ & $35(15.8)$ & $4.8(2.0,7.6)$ & $8(3.6)$ & $\begin{array}{l}8.1(4.8 \\
11.6)\end{array}$ \\
\hline RSV & $441(9.8 \%)$ & $4.4(2.9,8.3)$ & $71(16.1)$ & $4.0(2.1,8.0)$ & $23(5.2)$ & $\begin{array}{l}8.1(3.7 \\
25.5)\end{array}$ \\
\hline PIV & $368(8.2 \%)$ & $4.2(2.3,8.1)$ & $55(14.9)$ & $5.2(2.6,9.7)$ & $18(4.9)$ & $\begin{array}{l}10.8(5.0 \\
18.9)\end{array}$ \\
\hline HMPV & $363(8.1 \%)$ & $4.8(2.7,8.6)$ & $60(16.5)$ & $4.4(2.0,8.5)$ & $14(3.9)$ & $\begin{array}{l}12.0(8.6, \\
19.9)\end{array}$ \\
\hline $\mathrm{AV}$ & $93(2.1 \%)$ & $\begin{array}{l}5.1(2.7 \\
11.2)\end{array}$ & $21(22.6)$ & $\begin{array}{l}6.5(3.6 \\
13.6)\end{array}$ & $7(7.5)$ & $\begin{array}{l}38.0(11.2, \\
39.0)\end{array}$ \\
\hline $\mathrm{All}^{\mathrm{b}}$ & $4232(100 \%)$ & $4.2(2.3,7.8)$ & 609 (14.4) & $4.0(2.1,8.4)$ & $171(4.0)$ & $(5.0,19.8)$ \\
\hline
\end{tabular}

Abbreviations: LOS, length of stay; ICU, intensive care unit; IQR, interquartile range; Flu, influenza; $\mathrm{RV} / \mathrm{EV}$, rhinoviruses/ enteroviruses; CoV, coronavirus; RSV, respiratory syncytial virus; PIV, parainfluenza viruses; HMPV, human metapneumovirus; AV, adenovirus.

${ }^{a}$ Includes patients reported to have equivocal detection of influenza A.

${ }^{\mathrm{b}}$ Includes patients with more than one virus detected.

Figure Legends

Figure 1: Epidemiologic Curve of Coronaviruses from October 2017 - October 2019. The number of patients hospitalized with the human coronaviruses (CoV) 229E, HKU1, NL63 and OC43 during the study period are shown. Human coronavirus HKU1 was most common during the winter months of year 1 and human coronavirus $229 \mathrm{E}$ was most common during the winter months of year 2. Human coronavirus 229E was not detected until March 2018.

Figure 2a and 2b: Year 1 (Figure 2a) and Year 2 (Figure 2b) Comparative Population-based 
Incidence of Respiratory Viruses by Age Strata. The incidence of each virus per 100,000 persons (95\% confidence intervals) overall and by the three age strata 18-49, 50-64, 65-79, and $>80$ years of age are shown for year 1 and year 2. For both years and for every virus, the population-based incidence was highest for those $>80$ years of age.

\section{Hosted file}

Sieling et al. Figure 1.pdf available at https://authorea.com/users/357534/articles/498905comparative-incidence-and-burden-of-respiratory-viruses-associated-with-hospitalizationin-adults-in-new-york-city

\section{Hosted file}

Sieling et al. Figure 2.pdf available at https://authorea.com/users/357534/articles/498905comparative-incidence-and-burden-of-respiratory-viruses-associated-with-hospitalizationin-adults-in-new-york-city 